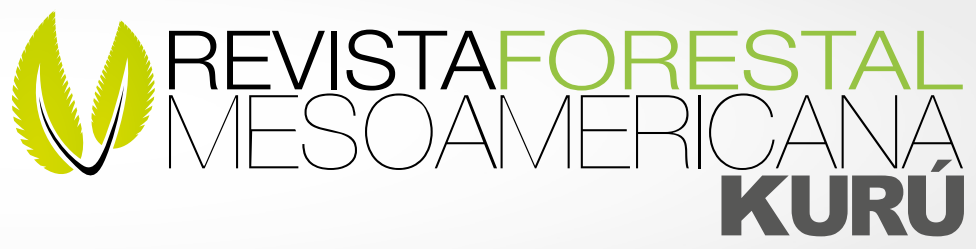

\section{Efecto inicial de los residuos de aprovechamiento forestal en el carbono de un suelo granítico de chile}

\author{
Edwin Antonio Esquivel-Segura ${ }^{1}$ \\ Rafael Alejandro Rubilar-Pons ${ }^{2}$ \\ Jorge Cancino-Cancino ${ }^{3}$
}

\section{Resumen}

Se determinó el impacto de la biomasa remanente postaprovechamiento en la concentración de carbono en los primeros $10 \mathrm{~cm}$ de suelo. Se ensayaron tres tratamientos de manipulación de residuos del aprovechamiento forestal aplicados post- aprovechamiento en una plantación forestal de Pinus radiata de 22 años: (a) remoción de los residuos del aprovechamiento de la superficie (X0), (b) testigo sin remoción ni adición de biomasa de residuos (X1), y (c) doble de la biomasa de residuos post- aprovechamiento (X2). Los contenidos de carbono se evaluaron mensualmente en los primeros $10 \mathrm{~cm}$ de suelo en el primer año post-tratamiento, mediante el método de pérdida de peso por ignición. Transcurridos 6 meses del inicio del experimento, la biomasa remanente del aprovechamiento fue de $2,7 \pm 1,8$; $37,4 \pm 3,3$ y $56,9 \pm 8,6 \mathrm{Mg} / \mathrm{ha}$ en $\mathrm{X0}, \mathrm{X} 1$ y X2 respectivamente. A pesar de la gran diferencia inicial en biomasa de residuos

\begin{abstract}
The aim of this study was to determine the impact of postharvest biomass remaining in the carbon concentration in the first $10 \mathrm{~cm}$ of soil. Three treatments were tested for handling harvest residues applied post-harvest forest to plantation forest of Pinus radiata in 22 years: (a) removal of harvest residues on the surface (X0), (b) control without removal or addition biomass residues (X1) and (c) twice the biomass of post-harvest residue (X2). Carbon contents were assessed monthly in the first $10 \mathrm{~cm}$ of soil in the first year post-treatment using the method of weight loss on ignition. After 6 months of the experiment, the remaining biomass harvest was $2.7 \pm 1.8,37.4 \pm 3.3$ and $56.9 \pm 8.6 \mathrm{Mg} / \mathrm{ha}$ in X0, X1 and X2 respectively. Despite the large initial difference in biomass residues remaining in treatment during the first year post-harvest, there was no difference in the carbon content in the soil between
\end{abstract}

1. Tecnológico de Costa Rica, Escuela de Ingeniería Forestal; Cartago, Costa Rica; edwinesquive/@itcr.ac.cr. Correspondencia. 2. Universidad de Concepción, Facultad de Ciencias Forestales, Cooperativa de Productividad Forestal; Concepción, Chile; rrubilar@ncsfnc.cfr.ncsu.edu

3. Universidad de Concepción, Facultad de Ciencias Forestales; Concepción, Chile; jcancino@udec.cl
Recibido: 10/09/2015 Aceptado: 28/09/2015 
remanente en los tratamientos, durante el primer año postaprovechamiento, no se evidenciaron diferencias en los contenidos de carbono en el suelo entre tratamientos. La reducción promedio del carbono del suelo fue un 68,2 \%, desde $67,7 \pm 12,5 \mathrm{Mg} / \mathrm{ha}$ (noviembre 2007) a 46,2 $\pm 4,4 \mathrm{Mg} /$ ha (octubre 2008).

Palabras clave: horizonte orgánico, residuos forestales, aprovechamiento forestal, carbono en suelo.

\section{Introducción}

En la generación de indicadores de manejo sostenible de suelos con plantaciones forestales se han empleado, criterios físicos, químicos y biológicos (Schoenholtz et al. 2000). Uno de esos indicadores ha sido la materia orgánica, que se conoce como todos los desechos orgánicos en el suelo que se encuentran en algún estado de descomposición (Sparks, 1995). Ha sido propuesta como, un buen indicador del manejo sustentable de este por su estrecha relación entre ésta y las propiedades físicas y químicas del suelo y son altamente dependientes del contenido de nutrientes y sus ciclos, infiltración y capacidad de almacenamiento de agua, erosión, evaporación, temperatura del suelo, cantidad de carbono acumulado, estructura, capacidad de intercambio de cationes; así también, constituye fuente de energía para microflora y microfauna del suelo (Kimmins, 1995; Tabatabai, 1996; Waring y Running, 1998, Fisher y Binkley, 1999; Powers 1999).

Los bosques forman un horizonte orgánico llamado también "piso forestal o mantillo", que se forma en la superficie de los horizontes del suelo. Esta es una de las mayores diferencias entre los suelos forestales y los dedicados a la agricultura. El horizonte orgánico superficial está conformado de biomasa de hojas y ramas caídas o por residuos del manejo propio del bosque, incluye los desechos de los aprovechamientos forestales y es uno de los componentes más dinámicos, complejos y reactivos. Su transformación y los productos de ésta causan cambios químicos, físicos y biológicos en el suelo subyacente. Tabatabai (1996), Fisher y Binkley (1999), proponen a la biomasa proveniente de los horizontes orgánicos como la fuente de energía para la biota del suelo.

La descomposición de la biomasa libera sustancias orgánicas que se lixivian y se integran desde el horizonte superior orgánico al suelo mineral. La descomposición de los desechos es llevada a cabo en gran medida por animales invertebrados, hongos, bacterias y la integración realizada principalmente por las lombrices. La velocidad a la que se descompone está relacionada con factores propios de la materia orgánica, tal como su misma treatments. The average reduction of soil carbon was $68.2 \%$, from $67.7 \pm 12.5 \mathrm{Mg} / \mathrm{ha}$ (November 2007) to 46.2 $\pm 4.4 \mathrm{Mg} / \mathrm{ha}$ (October 2008).

Key words: organic horizon, forest residues, forest harvest, carbon on soil.

composición, factores macro y microambientales y otros actores externos como el clima, fertilidad, drenaje, etc. Autores como, Kimmins (1995), Fisher y Binkley (1999) aseguraron que, la cantidad de biomasa del horizonte orgánico superficial, afecta la temperatura, humedad, aireación y por ende la cantidad de microorganismos presentes en el sitio encargados de la descomposición. La degradación de la materia se ve además afectada por la calidad de la biomasa aportada al suelo, porque cambia la relación entre el carbono y otros elementos nutricionales (Waring y Running, 1998). Por lo anterior se puede deducir que una manipulación de los aportes de materia orgánica en forma artificial influye en la velocidad de descomposición y afecta todos los factores relacionados con la materia orgánica en el suelo.

El aprovechamiento forestal extrae entre $50 \%$ y $77 \%$ de la biomasa aérea (Ximenes et al. 2008). La biomasa remanente se acumula en el suelo, influye en la temperatura, al disminuir la incidencia de rayos solares sobre el suelo (Powers, 1999), lo que aumenta la humedad del suelo y eventualmente favorece la descomposición de la materia orgánica. La acumulación de desechos orgánicos de forma excesiva y sin descomponer puede disminuir la velocidad de descomposición (Yanai et al. 2000) y provocar exceso de humedad en el suelo y acidificación, pobre crecimiento radicular y un estado nutricional deficiente al afectar los ciclos de varios nutrientes lo que reduciría el crecimiento vegetal esperado (Kimmins, 1995, Fleming et al. 2006). El incremento de materia orgánica puede inmovilizar mayor cantidad de nitrógeno y las propiedades químicas y biológicas del suelo, la productividad forestal y los ciclos del carbono por ejemplo (Yanai et al. 2000; Huang et al. 2008).

El carbono de los horizontes minerales es afectado por actividades antrópicas, particularmente en aquellos sitios donde se realiza extracción de residuos del aprovechamiento. Ussiri y Johnson (2007) encontraron cambios en la concentración de carbono en un suelo posterior al aprovechamiento, éstos retornaron a niveles

1. Cristian Higuera, Modelo Nacional de Simulación, Facultad de Ciencias Forestales, Universidad de Concepción. Comunicación personal. 
pre-aprovechamiento sólo después de 15 años. Los últimos autores mencionados concluyeron que existen cambios en el suelo como una reestructuración y reorganización de los materiales depositados en los horizontes orgánicos y que esto afecta horizontes minerales del suelo. En contraste a lo señalado Yanai et al. (2000) atribuyen los cambios del carbono del suelo a cambios en la composición del bosque, a las técnicas empleadas en el aprovechamiento forestal y a la velocidad de incorporación de los desechos en el suelo.

El tiempo de evaluación de los cambios en el carbono de suelo presenta algunas discrepancias entre autores, por un lado algunos como Mathers et al. (2003) y Sánchez et al. (2006) no encontraron diferencias significativas en la cantidad de carbono después de cinco años de duplicar la cantidad de materia orgánica que naturalmente habría quedado sobre el suelo post- aprovechamiento. Mathers et al. (2003) sugiere que al cambiar los niveles de materia orgánica sobre el suelo genera cambios en calidad y no en cantidad de carbono en los horizontes minerales. Por otra parte, Esquivel et al (2013) evidenciaron cambios en los niveles de carbono de plantaciones forestales establecidas post-aprovechamiento a los cuatro años de evaluación.

El objetivo de este estudio fue evaluar el efecto de la cantidad de biomasa proveniente de residuos del aprovechamiento forestal en las concentraciones de carbono en los primeros $10 \mathrm{~cm}$ del suelo y en los horizontes orgánicos. La hipótesis fue que una mayor acumulación de desechos aumenta el nivel de carbono en el suelo.

\section{Material y métodos}

Área de estudio. El ensayo fue establecido en la Región del Biobío, Chile a una latitud de $36^{\circ} 50^{\prime} \mathrm{S}$ y una longitud de $73^{\circ} 02^{\prime} \mathrm{W}$, a 100 m.s.n.m. Los meses más secos fueron de diciembre a febrero y la precipitación anual fue de $1912 \mathrm{~mm}$; la temperatura media anual fue de $12,6{ }^{\circ} \mathrm{C}$ (Observatorio Geodésico TIGO, 2008). El suelo del orden Alfisoles y suborden Xeralfs (USDA, 2006), pertenece a la serie San Esteban, de origen granítico, profundo (> 1,20 m), caracterizado por la susceptibilidad a la erosión y compactación de los horizontes superficiales. Análisis de laboratorio realizados a 36 muestras de suelo del área de estudio al inicio del ensayo, mostraron una textura arcillosa para el horizonte superficial (0 a $10 \mathrm{~cm}$ de profundidad), niveles máximos observados de materia orgánica del 15 $\%$ y una densidad aparente promedio de 1,13 $\pm 0,1 \mathrm{~g} / \mathrm{cm}^{3}$.

En marzo 2007 se realizó el aprovechamiento tradicional de una plantación de Pinus radiata D. Don de 22 años, el total de madera aprovechada para pulpaje fue de 220 $\mathrm{m}^{3 /}$ ha. Los ensayos se realizaron en agosto del mismo año una vez establecida una plantación de Eucalyptus globulus Labill, a un distanciamiento de $3 \mathrm{~m}$ entre hileras y 2 m entre plantas (1660 árboles/ha).

Diseño experimental. El diseño experimental fue de bloques completos al azar. La ubicación en los bloques se realizó en posiciones de alta, media y baja ladera. Los tres bloques fueron ubicados con la misma exposición de la ladera, con una pendiente promedio de $20 \%$ y una longitud inferior a $200 \mathrm{~m}$. Los tratamientos fueron aplicados en parcelas de $10 \mathrm{~m}$ x $10 \mathrm{~m}\left(100 \mathrm{~m}^{2}\right)$ para un área total de ensayo de $900 \mathrm{~m}^{2}(\mathrm{n}=3$ para cada tratamiento). Los tratamientos de manejo de residuos del aprovechamiento forestal fueron aplicados manualmente, con el objetivo de minimizar el impacto mecánico en el suelo. Los tratamientos fueron: (a) extracción total de la biomasa remanente del aprovechamiento (X0) (acículas, ramas delgadas (menores a $2,5 \mathrm{~cm}$ de diámetro), ramas gruesas (mayores de $2,5 \mathrm{~cm}$ de diámetro), conos, corteza y restos de fustes); (b) tratamiento testigo donde no se realizaron aportes ni remociones de biomasa (X1); y (c) doble incorporación de biomasa de residuos del aprovechamiento, distribuyéndola homogéneamente sobre el suelo (X2).

Con el fin de evitar la interferencia de la vegetación competidora emergente que pudiese afectar las determinaciones de carbono y el crecimiento de la plantación establecida, en octubre de 2007 se realizó un control químico con Glifosato $(2,0 \mathrm{~kg} / \mathrm{ha})$, Ixosaflutole (350 g/ha) y Silwet L 77 AG (100 cc/ha), en febrero de 2008 se realizó un segundo control químico con Glifosato $(2,5$ $\mathrm{kg} / \mathrm{ha})$, Triclopir (0,5 I/ha) y Simazina (3 I/ha) y en octubre de 2008 se realizó un tercer control con Glifosato $(2,0$ kg/ha). Desde enero de 2008 hasta el 2009 se realizó un control manual de malezas para evitar regeneración abundante de especies como: mora (Rubus ulmifolius Schott), retamilla (Teline monspessulana (L.) K. Koch), quila (Chusquea quila Kunth), quilo (Muehlenbeckia hastulata (J.E. Sm.) Johnst), maqui (Aristotelia chilensis (Mol.) Stuntz).

Muestreo de biomasa de residuos remanentes de aprovechamiento. En febrero de 2008, a 6 meses de la aplicación de los tratamientos, se realizó la primera determinación de los contenidos de biomasa remanente post- aprovechamiento en cada uno de los tratamientos. Una segunda determinación fue realizada un año después en febrero de 2009. Para ambas determinaciones, en cada unidad experimental, fueron localizadas aleatoriamente cuatro áreas de 0,066 $\mathrm{m}^{2}(0,29 \mathrm{~m}$ de diámetro) (submuestras) y en ellas se recolectaron todos los componentes de biomasa de residuos remanentes en el sitio: ramas gruesas (mayores a $2,5 \mathrm{~cm}$ de diámetro), ramas delgadas (menores de $2,5 \mathrm{~cm}$ de diámetro), conos, corteza, material enterrado (ramas o raíces degradadas presentes en un horizonte mineral), los horizontes orgánicos Oi (conformado por hojas, acículas y ramillas delgadas frescas y ligeramente descompuestas), Oe 
(fragmentos donde el proceso de descomposición había avanzado claramente, pero aún se puede identificar el origen del material) y Oa (compuesto por materiales orgánicos altamente fragmentados y degradados, donde es imposible reconocer el origen del material ya que es un material amorfo) (Waring y Running, 1998; Fisher y Binkley, 1999) según la metodología utilizada por Rubilar (2003). Para cada submuestra se obtuvo una muestra compuesta $(n=5)$ del suelo a dos profundidades ( 0 a 5 $\mathrm{cm}$ y 5 a $10 \mathrm{~cm}$ ), tomadas con barreno. Si en la muestra de suelo aparecía biomasa enterrada fue separada y analizada por separado.

Muestreo de materia orgánica mensual en el suelo. Muestras compuestas (12 submuestras) mensuales de los primeros $10 \mathrm{~cm}$ de suelo fueron colectadas en cada unidad experimental a partir de noviembre de 2007. Cada unidad experimental fue dividida en 12 cuadrantes a fin de captar la variabilidad existente y el muestreo de suelo se realizó en cada cuadrante mediante un barreno helicoidal de $3 \mathrm{~cm}$ de diámetro, previa remoción de los horizontes orgánicos en cada punto de muestreo. Cada cuadrante se muestreó aleatoriamente, se evitó muestear los bordes de parcela y cada punto de muestreo fue marcado para evitar muestrearlo repetidamente en los meses siguientes.

Análisis de laboratorio. Para determinar el peso seco de la biomasa $( \pm 0,1 \mathrm{~g})$ de ramas gruesas, ramas delgadas, conos, corteza, material enterrado y horizontes orgánicos Oi, Oe y Oa se secó a $105^{\circ} \mathrm{C}$ por 24 horas cada biomasa por separado. Con el fin de corregir contaminación de los horizontes orgánicos con el suelo mineral se calcinó a $450{ }^{\circ} \mathrm{C}$ por 24 horas y se determinó su peso con una incertidumbre de $( \pm 0,0001 \mathrm{~g})$.

Las muestras de suelo se secaron al aire por al menos 24 horas, este secado al aire inició el mismo día que fueron obtenidas. Posteriormente se tamizaron a $2 \mathrm{~mm}$ (mesh ASTM No 10) y se determinó los pesos de grava (partículas $>2 \mathrm{~mm}$ ), biomasa de raíces y suelo $(<2 \mathrm{~mm})$ $( \pm 0,1 \mathrm{~g})$.

La determinación de materia orgánica en el suelo fue realizada mediante el método de pérdida de peso por ignición WLOI (Weight-Loss-On-Ignition) (Magdoff, 1996). Para ello, una alícuota de $10 \mathrm{~g}$ de suelo $( \pm 0,0001$ g) se secó por 24 horas en horno a $105^{\circ} \mathrm{C}$ para obtener el peso seco del suelo ( $\pm 0,0001 \mathrm{~g}$ ). Posteriormente, las muestras fueron calcinadas en una mufla a $450{ }^{\circ} \mathrm{C}$ por 24 horas y pesadas ( $\pm 0,0001 \mathrm{~g}$ ) para obtener el peso calcinado. Previo al pesaje se permitió que las muestras alcanzaran temperatura ambiente en un desecador. Todos los análisis de cada muestreo fueron realizados en duplicado. El contenido de materia orgánica (MO \%) se determinó mediante la fórmula (1)

$$
\mathrm{MO}(\%)=\frac{\mathrm{PSS}_{105^{\circ} \mathrm{C}}-\mathrm{PSC}_{450^{\circ} \mathrm{C}}}{\mathrm{PSS}_{105^{\circ} \mathrm{C}}} * 100
$$

Donde

$\mathrm{PSS}_{105^{\circ} \mathrm{C}}$ : peso seco del suelo $(\mathrm{g})$

$\mathrm{PSC}_{450^{\circ} \mathrm{C}}$ : peso calcinado (g)

Las determinaciones de carbono en suelo se escalaron con el porcentaje de materia orgánica mediante la fórmula [2] con un valor promedio de $50 \%$ de carbono en la materia orgánica acorde a Tabatabai (1996).

$$
\text { Carbono }=\frac{\mathrm{VS} * \mathrm{Da} * \mathrm{MO} \%}{2}
$$

Donde

VS : Volumen de suelo $\left(\mathrm{m}^{3} / \mathrm{ha}\right)$

$\mathrm{Da}$ : Densidad aparente $\left(\mathrm{Mg} \mathrm{m}^{-3}\right)$

MO : Materia orgánica (\%)

La densidad aparente se determinó mediante 3 submuestras por unidad experimental a profundidades de 0 a $5 \mathrm{~cm}$ y 5 a $10 \mathrm{~cm}$ tomadas con un barreno para muestreo de densidad aparente con cilindros de $5 \mathrm{~cm}$ de diámetro. Se determinó el volumen del cilindro y el peso seco del suelo a $( \pm 0,1 \mathrm{~g})$.

Análisis de nitrógeno. Mediante el método de semimicro Kjeldahl (Longeri et al. 1979) se analizó químicamente el contenido de nitrógeno a las muestras $(n=27)$ obtenidas en los meses de noviembre de 2007 , abril y septiembre de 2008.

Análisis estadístico. Los datos se sometieron a un análisis de homocedasticidad mediante la prueba de Bartlett a los residuales de las variables: biomasa de residuos, carbono de suelo y nitrógeno total y se aplicó la prueba de Shapiro-Wilks para verificar su normalidad con el software estadístico SAS Versión 9.1 (SAS Institute Inc, 2000). Las medias se compararon mediante la prueba de Tukey con un $95 \%$ de confianza. Para datos que no presentaron distribución normal u homocedasticidad se utilizó el test de Friedman con un $95 \%$ de confianza mediante la comparación de medias con el software estadístico SAS Versión 9.1 (SAS Institute Inc, 2000).

Se hizo una evaluación gráfica de modelos con el programa JMP 7 (SAS Institute Inc, 2008), para describir el comportamiento en el tiempo del contenido de carbono del suelo seleccionándose el modelo de decaimiento cinético de primer orden. Al modelo se le incorporó un modelo sinusoidal que emuló ese comportamiento detectado en las observaciones. Se buscó explicar este comportamiento en base a variables ambientales: temperatura media mensual, número de horas mensuales con temperaturas promedio entre 0 y $10{ }^{\circ} \mathrm{C}, 10$ y $20^{\circ} \mathrm{C}$ y mayores de $20{ }^{\circ} \mathrm{C}$, humedad relativa mínima diaria promediada mensualmente, precipitación mensual ( $\mathrm{mm})$ y tiempo transcurrido desde la primera medición 
en días julianos (días desde que se realiza la primera determinación de suelo). Posteriormente, se modeló la dinámica del carbono en el suelo por tratamiento para finalmente realizar una comparación entre los modelos obtenidos. El modelo utilizado fue de tipo:

$$
Y=\beta_{0}+\beta_{1} e^{\left(\beta_{2} x\right)}+\beta_{3} \operatorname{seno}((x-90) / 57,29577951)
$$

Donde

$\mathrm{Y}=$ carbono en suelo $(\mathrm{Mg} / \mathrm{ha})$

$\mathrm{X}=$ tiempo en días post-tratamiento

$\beta_{0}, \beta_{1}, \beta_{2}$, y $\beta_{3}=$ son parámetros a estimar.

\section{Resultados}

Análisis de residuos del aprovechamiento postaplicación de tratamientos en el horizonte orgánico. La biomasa residual en el suelo del tratamiento $\mathrm{XO}$, en febrero de 2008, i.e. 6 meses después de establecido el experimento difirió $(P=0,001)$ del tratamiento $\mathrm{X} 2$ (figura 1), pero no hubo diferencias entre $\mathrm{X} 1$ y X2. Estas diferencias no cambiaron al evaluar los horizontes no manipulados Oe y $\mathrm{Oa}$. La diferencia promedio entre los tratamientos X1 y X2 y el tratamiento X0 fue de $45,4 \mathrm{Mg} /$ ha para la biomasa manipulada.

En febrero de 2008, la biomasa de conos y corteza no presentó homocedasticidad. La comparación evidenció diferencias en la biomasa de conos y corteza $(P=0,05)$ los valores más altos fueron los obtenidos en el tratamiento X2 comparándolo con X0 (Figura 2). Las ramas gruesas $(P=0,0329)$, ramas delgadas $(P=0,0040)$ y horizontes Oi $(P<0,001)$ y Oe $(P=0,0299)$ presentaron valores más altos en el tratamiento X2 en comparación con el X0. Por el contrario, no se encontraron diferencias en la cantidad de residuos para los materiales enterrados $(P=0,4426)$ ni para el horizonte Oa $(P=0,4249)$ (Figura 2$)$.

En febrero de 2009, las ramas delgadas y el material enterrado no presentaron homocedasticidad. Se encontró diferencias significativas entre los tratamientos en las cantidades de biomasa de ramas delgadas $(P<0,001)$ y en el horizonte orgánico Oi $(P=0,0385)$. Las demás estructuras no evidenciaron diferencias significativas (conos $P=0,2498$; corteza $P=0,6440$; ramas gruesas $P=0,3614$; material enterrado $P=0,1482$; Oe $P=0,2331$; Oa $P=0,8661$ ) (Figura 2).

Entre los años 2008 y 2009 no se registraron cambios en los contenidos de biomasa de las estructuras que fueron manipuladas (ramas gruesas, ramas delgadas, conos, corteza y horizonte orgánico $\mathrm{Oi}$ ) en ninguno de los tratamientos $(\mathrm{X} 0 P=0,7716, \mathrm{X} 1 P=0,2158$ y X2 $P=0,2011)$. $\mathrm{Al}$ analizar los contenidos totales de biomasa incluyendo las estructuras manipuladas y no manipuladas, tampoco se encontraron diferencias para los tratamientos $\mathrm{X} 0(P=$ $0,1527)$ y $\mathrm{X} 1(P=0,5921)$. Sin embargo, se evidenció una

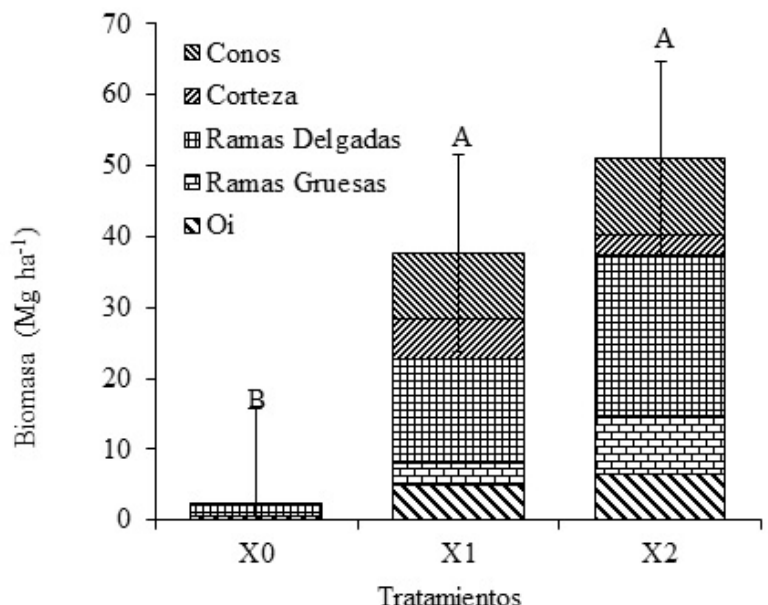

Figura 1. Biomasa de residuos de cosecha agrupado por componente en cada tratamiento. $\mathrm{X} 0$, remoción de biomasa de residuos; $\mathrm{X} 1$, testigo (residuo natural de la cosecha) y X2, doble de la cantidad de biomasa de residuos natural en febrero 2008. Letras distintas indican diferencias significativas (Test de Tukey $a=0,05$ ). Barras de error corresponden a intervalos para el test de Tukey para la biomasa por tratamiento.

Figure 1. Yield residues biomass grouped by component in each treatment. X0, waste biomass removal; $\mathrm{X} 1$, control (natural crop residue) and X2, double amount of natural waste biomass in February 2008. Different letters indicate significant differences (Tukey test $a=0,05$ ). Error bars correspond to Tukey test's intervals for biomass treatment.
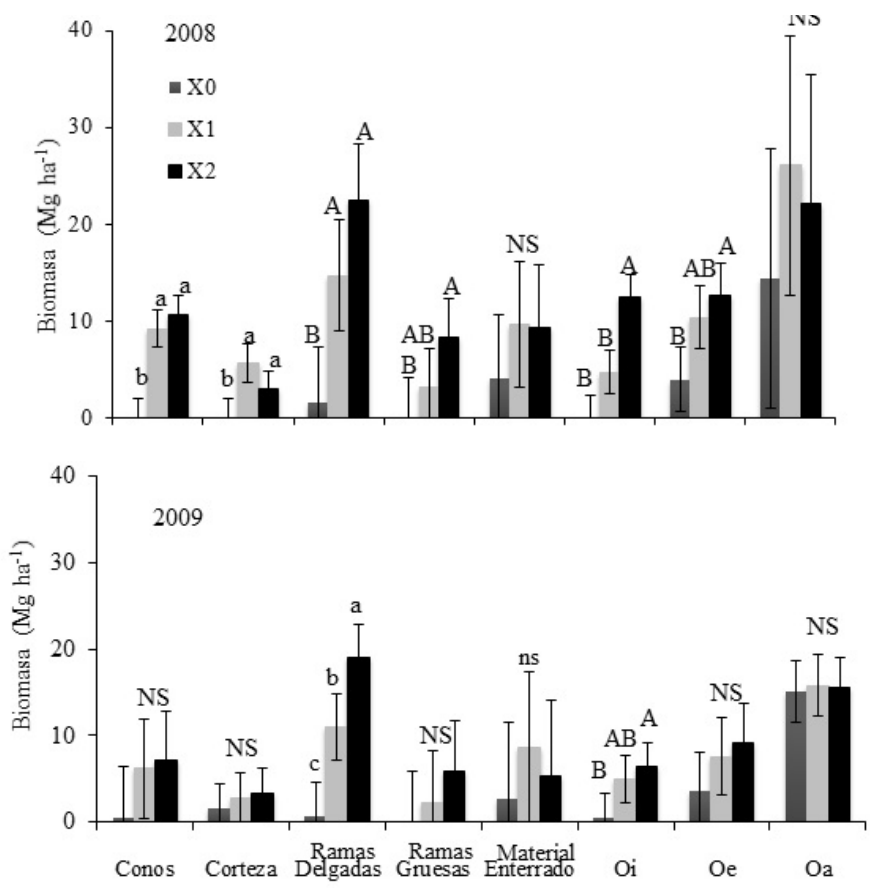

Figura 2. Biomasa del horizonte orgánico para cada tratamiento: $\mathrm{X} 0$, remoción de biomasa de residuos; $\mathrm{X} 1$, testigo (residuo natural de la cosecha) y X2, doble de la cantidad de biomasa de residuos, diferenciado por componentes muestreados para febrero 2008 y 2009. Letras distintas indican diferencias significativas (en mayúsculas Test de Tukey, $a=0,05$; en minúsculas Test Freidman, $a=0,05$ ). Barras de error corresponden a intervalos para el Test de Tukey o Freidman, según corresponda.

Figure 2. Organic horizon biomass for each treatment: $X 0$, residues biomass removal; $\mathrm{X} 1$, control (natural harvest residue) and $\mathrm{X} 2$, double amount of residues biomass, differentiated by sampled components in February 2008 and 2009. Different letters indicate significant differences (Tukey test in capital letters, $a=0,05$; Freidman test in lowercases, $a=$ 0.05). Error bars correspond to intervals for the Tukey or Freidman test. 


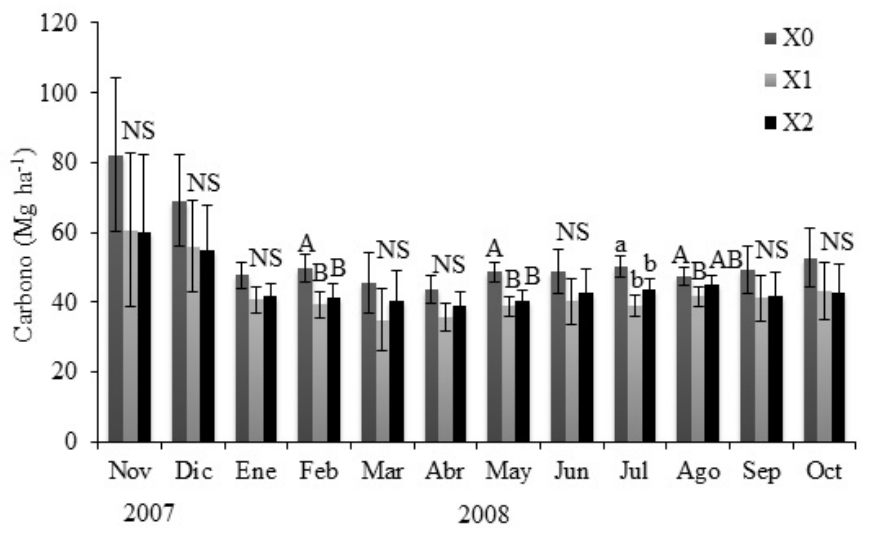

Figura 3. Carbono del suelo $(0-10 \mathrm{~cm})$ a lo largo del tiempo para los tratamientos X0 (remoción de la biomasa de residuos), X1 (testigo) y X2 (doble cantidad de biomasa de residuos). Letras distintas indican diferencias significativas (en mayúsculas Test de Tukey, $a=0,05$; en minúsculas Test Freidman, $a=0,05)$. Barras de error corresponden a intervalos para el Test de Tukey o Freidman, según corresponda.

Figure 3. Soil carbon $(0-10 \mathrm{~cm})$ over time for treatments. X0 (residues biomass removal), X1 (control) and X2 (double amount of residues biomass). Different letters indicate significant differences (Tukey test in capital letters, $a=0,05$; Freidman test in lowercases, $a=0.05$ ). Error bars correspond to intervals for the Tukey or Freidman test.

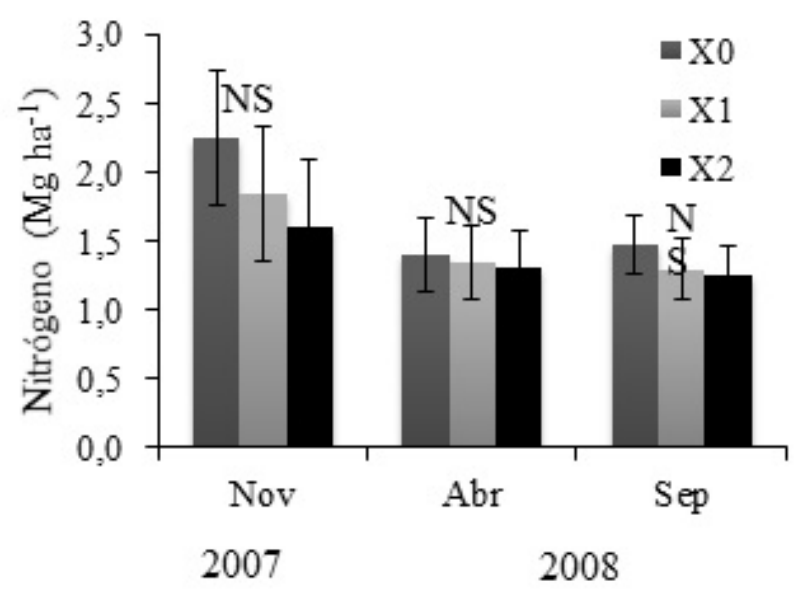

Figura 5. Contenidos de nitrógeno $\mathrm{Mg} / \mathrm{ha}$ para los primeros $10 \mathrm{~cm}$ de suelo en los tratamientos: $\mathrm{X} 0$, remoción de la biomasa de residuos; $\mathrm{X} 1$, testigo; X2, doble cantidad de biomasa de residuos. No existen diferencias significativas (Test de Tukey $\alpha=0,05$ ). Barras de error corresponden a intervalos Tukey.

Figure 5. Nitrogen content $\mathrm{Mg}$ ha- 1 for the first $10 \mathrm{~cm}$ of soil in treatments: $\mathrm{X} 0$, residues biomass removal, $\mathrm{X} 1$, control; and $\mathrm{X} 2$, double amount of residues biomass. No significant differences (Tukey test, $\alpha=$ 0,05). Error bars correspond to intervals Tukey.

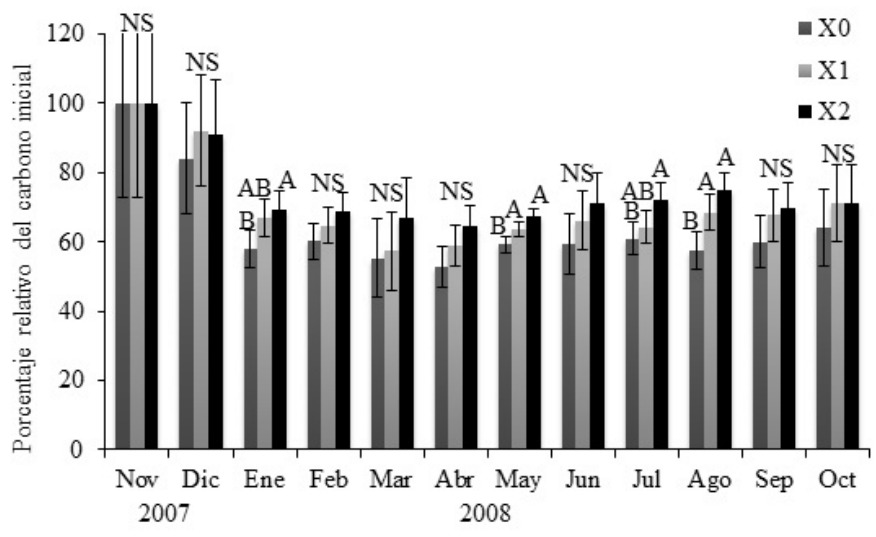

Figura 4. Porcentaje relativo al valor inicial del carbono en el suelo a lo largo del tiempo para los tratamientos: X0, remoción de la biomasa de residuos; X1, testigo y X2, doble cantidad de biomasa de residuos. Letras distintas indican diferencias significativas (Test de Tukey $a=$ $0,05)$. Barras de error corresponden a intervalos para el test de Tukey.

Figure 4. Percentage relative to initial value of soil carbon over time for treatments: X0, waste biomass removal, X1, control and X2 double amount of waste biomass. Different letters indicate significant differences (Tukey test, $\alpha=0,05$ ). Error bars correspond to intervals for the Tukey test.

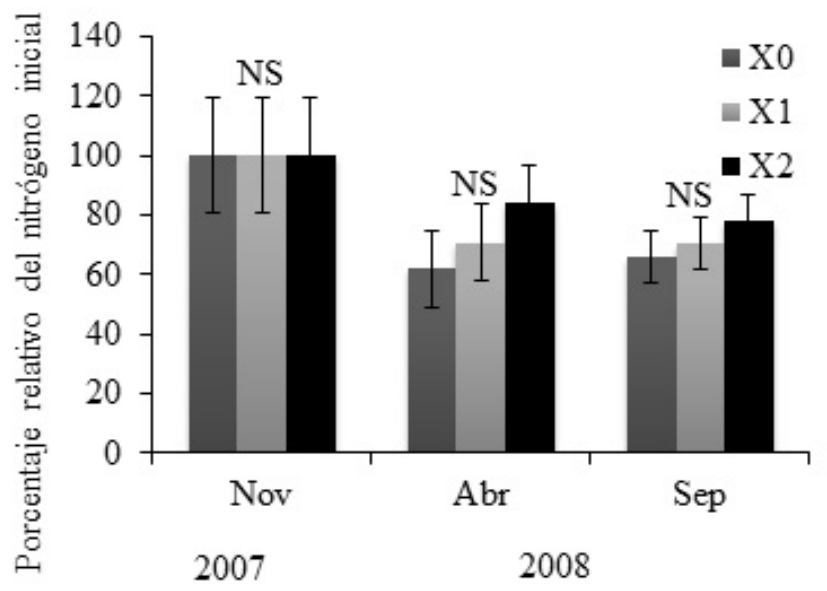

Figura 6. Contenidos de nitrógeno total relativos a la cantidad inicial. X0, remoción de la biomasa de residuos; X1, testigo y X2, doble cantidad de biomasa de residuos. No se encontraron diferencias significativas (Test de Tukey $a=0,05$ ) Barras de error corresponden a intervalos para el test de Tukey.

Figure 6. Total nitrogen content relative to the initial amount. XO, waste biomass removal; $\mathrm{X} 1$, control; and $\mathrm{X} 2$, double amount of waste biomass. No significant differences (Tukey test, $a=0,05$ ). Error bars correspond to Tukey's intervals. 
Cuadro 1. Modelado del carbono del suelo $(0-10 \mathrm{~cm})$; 1) modelo de decaimiento cinético, 2) incorporación de una variación estacional, 3) modelo para el tratamiento X0, 4) modelo para las tratamientos X1 y X2. Para cada modelo los parámetros respectivos.

Table 1. Soil carbon modeling $(0-10 \mathrm{~cm})$; 1) kinetic decay model, 2) incorporating seasonal variation, 3) model for treatment $\mathrm{X} 0$, 4) model for treatments X1 and X2. The respective parameters are presented for each model.

\begin{tabular}{|c|c|c|c|c|}
\hline \multirow{2}{*}{ Modelo } & \multicolumn{4}{|c|}{ Parámetros } \\
\hline & $\beta 0$ & $\beta 1$ & $\beta 2$ & $\beta 3$ \\
\hline 1) $Y=\beta 0+\beta 1$ e ( $\beta 2 x)$ & 42,8199 & 62,1799 & $-0,0286$ & \\
\hline 2) $Y=\beta 0+\beta 1$ e $(\beta 2 x)+\beta 3 \operatorname{seno}((x-90) /(57,29))$ & 43,0781 & 50,8069 & $-0,0262$ & $-2,9582$ \\
\hline 3) $Y=\beta 0+\beta 1$ e $(\beta 2 x)+\beta 3$ seno $((x-90) /(57,29))$ & 48,1486 & 76,6959 & $-0,0285$ & $-3,0350$ \\
\hline 4) $Y=\beta 0+\beta 1$ e $(\beta 2 x)+\beta 3 \operatorname{seno}((x-90) /(57,29))$ & 40,5410 & 38,44559 & $-0,0245$ & $-2,9253$ \\
\hline
\end{tabular}

Todos los parámetros son significativos con un nivel de significancia de $\mathrm{P}<0,0001$

$\mathrm{Y}=$ Nivel de carbono

$\mathrm{X}=$ tiempo

disminución significativa de la biomasa entre los años 2008 y 2009 en el tratamiento X2 $(P=0,0175)$. Al analizarlo por estructuras esta disminución sólo fue significativa en la biomasa de los horizontes orgánicos Oi $(P=0,0313)$ y Oe $(P=0,0491)$. La biomasa de conos, corteza, ramas delgadas, ramas gruesas, material enterrado y horizonte Oa no presentaron variaciones entre ambos años (conos $P=0,5922$; corteza $P=0,9683$; ramas delgadas $P=$ 0,5210 ; ramas gruesas $P=0,5381$; material enterrado $P$ $=0,4951$; y horizonte Oa $P=0,0935$ ).

Dinámica del carbono. Los mayores descensos en los contenidos de carbono en el suelo se dieron en el periodo pre-estival el cual fue de 46,2 $\pm 4,4 \mathrm{Mg} / \mathrm{ha}$, valor equivalente a $68 \%$ de los contenidos de carbono del mes de noviembre (primer muestreo). Los contenidos de carbono por tratamiento fueron comparados por mes, encontrándose diferencias significativas para los meses de marzo $(P=0,0494)$ y julio $(P=0,004)$. Al comparar los contenidos de carbono por tratamiento mes a mes, se observó un descenso entre los meses de noviembre a enero en los contenidos de carbono, para luego mantenerse sin variaciones entre los meses de febrero a octubre. Al comparar cada mes los contenidos de carbono del suelo no se evidenciaron diferencias entre los tratamientos (Figura 3).

Si se toma en cuenta como un $100 \%$ la primera medición de carbono en el suelo, la disminución que presentan los tratamientos en los diferentes meses no difiere significativamente, con un valor promedio de $64,2 \%$ del carbono original. Sólo para el mes de julio existió diferencia, donde el X2 llegó a 71 \% y los demás tratamientos a $62,5 \%$ del nivel originalmente medido en noviembre (Figura 4).

Dinámica del nitrógeno total. En la determinación de los niveles de nitrógeno total para los meses de noviembre2007,

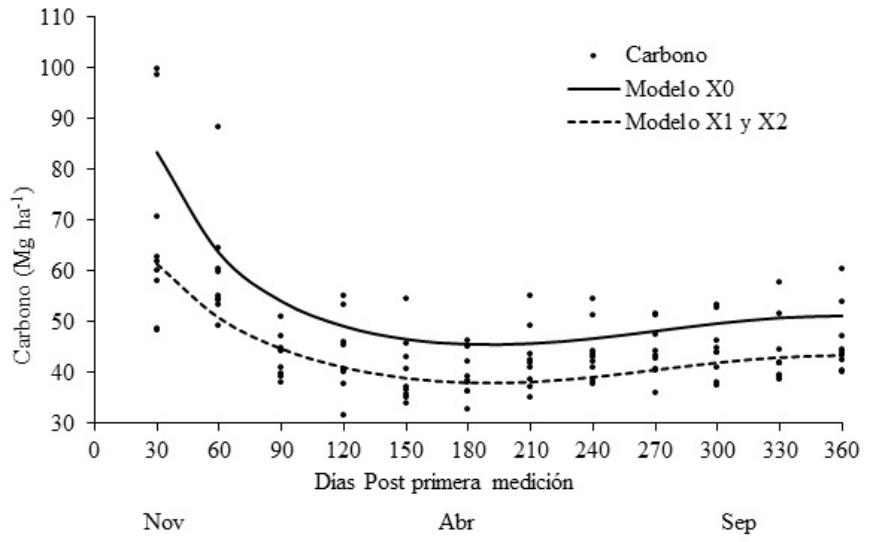

Figura 7. Modelado de la dinámica del carbono en el primer año postcosecha con $Y=\beta 0+\beta 1$ e $(\beta 2 x)+\beta 3$ seno $((x-90) /(57,29))$ ( $Y$ : carbono en suelo mineral (Mg/ha); $x$ : días Post-tratamientos; $\beta 0, \beta 1, \beta 2$ y $\beta 3$ parámetros de los modelos).

Figure 7. Carbon dynamics modeling in the first year post- harvest with $Y=\beta 0+\beta 1$ e $(\beta 2 x)+\beta 3$ seno $((x-90) /(57,29))(Y$ : carbon in the mineral soil (Mg/ha); $\mathrm{x}$ : post-treatments days; $\beta 0, \beta 1, \beta 2$ y $\beta 3$ model parameters).

abril y septiembre 2008, no se evidenciaron diferencias en los contenidos ni en la dinámica de nitrógeno de cada tratamiento. Del mismo modo los niveles de nitrógeno no variaron entre tratamientos (Figura 5).

Al analizar la dinámica del nitrógeno en términos relativos al valor original se encuentran únicamente diferencias entre los niveles de X0 $(P=0,0204)$ y X2 $(P=0,0030)$, y no se encontraron diferencias para los valores de $\mathrm{X} 1(P=0,1525)$, tampoco existen diferencias entre los tratamientos.

Modelado de la dinámica del carbono en el suelo. El ajuste del modelo contempló tanto el descenso en el carbono de los primeros tres meses de iniciado el muestreo mediante un componente de decaimiento cinético, como las variaciones de los contenidos de 
carbono en el suelo mediante la función seno. El modelo consiguió explicar el 84,7 \% de la variabilidad de los datos; la raíz del cuadrado medio residual evidenció un error promedio de 8,9 Mg/ha de carbono.

Al modelar la dinámica del carbono para cada uno de los tres tratamientos por separado y realizar una prueba $\mathrm{F}$ (Weisberg 1985), se detectó que entre los modelos obtenidos hay evidencias que al menos un modelo es diferente $(F=2,3>$ $\left.F_{a=0,05 ; 8 ; 107}=2,10\right)$. Los tratamientos $X 1$ y $X 2$ no presentaron diferencias entre sí $\left(F=0,2<F_{\mathrm{a}=0,05 ; 4 ; 7}=2,53\right)$. El modelo del tratamiento $\mathrm{X} 0$ es diferente a los otros tratamientos (con X1, $F=3,0>F_{\mathrm{a}=0,05 ; 4 ; 71}=2,53$; con $\left.X 2, F=2,6>F_{\mathrm{a}=0,05 ; 4 ; 71}=2,53\right)$.

\section{Discusión}

Dinámica de los residuos del aprovechamiento sobre el suelo. Este sitio registró una producción de $220 \mathrm{~m}^{3} /$ ha y, la cantidad de biomasa que aportó el aprovechamiento al suelo para diferentes estructuras: ramas, follaje y corteza, concuerda con estimaciones hechas por Gerding y Schlatter (1999), Merino et al. (2003) y Merino et al. (2005) tomando en cuenta que un aprovechamiento tradicional cosecha entre 50 y $77 \%$ del total de biomasa del sitio (Ximenes et al. 2008). Es esta biomasa residual la que es sujeta a ser extraída en un aprovechamiento de residuos y es lo que justamente el tratamiento XO emula, un aprovechamiento de este tipo que extrae totalmente ramas y restos del fuste. En ese tipo de aprovechamiento, frecuentemente queda biomasa que no se extrae, que en este caso correspondió a materiales enterrados y los horizontes orgánicos Oe (fragmentos donde el proceso de descomposición había avanzado claramente, pero aún se puede identificar el origen del material) y $\mathrm{Oa}$ (compuesto por materiales orgánicos altamente fragmentados y degradados, donde es imposible reconocer el origen del material ya que es un material amorfo). La forma en que se aplicaron los tratamientos minimizó el impacto en el suelo y los horizontes $\mathrm{Oe}$ y $\mathrm{Oa}$ que no fueron manipulados, es por esto que el horizonte $\mathrm{Oa}$ y los materiales enterrados no presentaron diferencias entre los tratamientos.

Sin embargo, a seis meses de la aplicación se observó una diferencia significativa entre los tratamientos X0 y X2, donde siempre lo valores en los contenidos de biomasa de ramas delgadas, ramas gruesas y el horizonte $\mathrm{Oi}$ fueron mayores en el X2 que en el X0. Estas diferencias fueron provocadas por la aplicación de los tratamientos, lo que demuestra que los aprovechamientos de residuos impactaron la cantidad de biomasa residual de un aprovechamiento. En el horizonte Oe se observó un aumento de biomasa en X2 a pesar de no haber sido manipulado. Lo anterior podría deberse a que la dinámica de descomposición, podría haber sido afectada por la composición y el tamaño del material (Brady y Weil, 2002).
Cabe destacar que los seis meses transcurridos desde la aplicación de los tratamientos hasta la primera evaluación de la biomasa remanente acumulada sobre el suelo, en apariencia fue insuficiente para que se realizara una descomposición de la biomasa a tal grado que pudiera afectar los contenidos en el horizonte $\mathrm{Oa}$, pero sí se provocó diferencias en Oe. Este horizonte a pesar de no ser removido en la aplicación de los tratamientos, presentó diferencias en la cantidad de biomasa entre X0 y X2, en este último fue mayor; se puede interpretar que a 6 meses de la aplicación de los tratamientos la descomposición de la biomasa del horizonte Oi (conformado por hojas, acículas y ramillas delgadas frescas y ligeramente descompuestas) habría afectado los contenidos en el horizonte Oe.

Al comparar la dinámica de cada tratamiento entre los años 2008 y 2009, no se evidenciaron diferencias entre los contenidos de residuos manipulados. Pero al comparar todos los horizontes, manipulados y no manipiulados, el descenso de la biomasa total de residuos fue significativo únicamente en el tratamiento X2. Cabe destacar que, el contenido de residuos sobre el suelo en el tratamiento X0 para el año 2009, supera los contenidos mínimos recomendados de entre 22 y 36 $\mathrm{Mg} / \mathrm{ha}$ para mantener un suelo biológicamente saludable (Jurgensen et al. 1997).

Carbono del suelo. El contenido de carbono en el suelo sufrió una caída entre noviembre de 2007 y enero de 2008 , posiblemente debido a que las temperaturas en este periodo fueron mayores a los meses invernales. La dinámica del carbono en los diferentes tratamientos no difirió estadísticamente; para los meses de febrero a julio el nivel de carbono en suelo se mantuvo sin diferencias, debido posiblemente a la falta de precipitaciones que permitieran condiciones de humedad adecuadas para aumentar la descomposición. En el mes de agosto, el tratamiento X0 continuó constante y el X2 presentó un leve aumento, equivalente al aumento del testigo. Sin embargo, estas pequeñas diferencias no lograron ser significativas por lo que a 12 meses de la aplicación de los tratamientos, y a pesar de evidenciar una diferencia del material sobre el horizonte orgánico, no se observaron diferencias significativas en los contenidos de carbono. Estos resultados concuerdan con los reportados por Kabzems y Haeussler (2005) y Sánchez et al. (2006) que no encontraron diferencias entre los contenidos de carbono en el suelo para los cinco primeros años después de la aplicación de los tratamientos de remoción y adición de residuos de aprovechamientos.

A pesar de lo anterior y como se puede observar en los resultados todos los tratamientos presentaron una caída en los niveles de carbono, esta caída cuantificada en megagramos o toneladas métrica por hectárea $(\mathrm{Mg} / \mathrm{ha})$ en los primeros $10 \mathrm{~cm}$ de suelo según datos entregados por el Modelo Nacional de Simulación de Pino Radiata, 
de la Facultad de Ciencias Forestales, es equivalente al carbono acumulado en la biomasa aérea de una plantación de Pinus radiata D. Don de 11 años en este sitio, catalogado como un sitio de nivel medio para la producción de $P$. radiata para la zona de Concepción.

Esta caída en los niveles de carbono en un corto plazo podrían también ser atribuidos a las especies utilizadas (del género Pinus y Eucalyptus), las cuales tiene una amplia distribución geográfica en el Continente Americano, por los efectos que las mismas podrían estar teniendo a nivel de calidad de materia orgánica aportada al suelo.

Efectos del aprovechamiento. Entre febrero de 2008 y 2009, no hubo diferencias significativas entre los niveles de carbono del suelo y biomasa del horizonte $\mathrm{Oa}$ a pesar que este horizonte perdió más biomasa entre los dos años evaluados. El horizonte Oa, presentó los compuestos orgánicos más degradados que pueden ser incorporados al suelo más rápidamente. Estos se incorporan en forma de coloides, solubles y la fracción más pequeña que se incorpora al suelo en forma de sólidos (Fisher y Binkley, 1999). Esto sugiere que la disminución de carbono en el suelo en el primer año postaprovechamiento fue un efecto del aprovechamiento y no de los tratamientos aplicados. Powers et al. (2005) encontraron diferencias en el carbono del suelo en profundidades de hasta $20 \mathrm{~cm}$ entre los tratamientos de adición y remoción de biomasa entre los 5 y 10 años posteriores a la aplicación del tratamiento, por lo que estos resultados podrían demostrar que el tiempo transcurrido es muy pequeño para obtener un efecto en el carbono del suelo. La disminución del carbono en el suelo en los primeros meses puede ser atribuida a la descomposición de las raíces finas y micorrizas (Powers et al. 2005), que dejan de cumplir su función metabólica y por ende aportar carbono al suelo.

Yanai et al. (2003) sugieren que múltiples factores podrían estar correlacionados con los cambios de carbono en el suelo; la vegetación, exposición, pendiente, tipo de suelo y clima son factores que podrían alterar los resultados (Yanai et al. 2000). En este sitio se tuvieron condiciones de exposición, pendiente, tipo de suelo y clima similares entre los tratamientos, por lo que los cambios pueden atribuirse a un efecto directo o indirecto post-aprovechameinto en la descomposición del carbono lábil del suelo. Merino et al. (1998) y Usiri y Johnson (2007) concuerdan con los cambios que ocurren en el descenso de la materia orgánica del suelo y en consecuencia del carbono, y atribuyen este efecto a cambios en la calidad de la materia orgánica.

Nitrógeno. El conocimiento de la dinámica que el nitrógeno post-aprovechamiento y el efecto que causan las extracciones de la biomasa es de suma importancia para los procesos silvícolas y regenerativos de un área (Vitousek, 1985). En este estudio, en el primer año, los contenidos de nitrógeno total no fueron diferentes entre los tratamientos posiblemente por el poco tiempo transcurrido. Brais et al. (2002), en suelos arcillosos, encontraron diferencias en los contenidos de nitrógeno total producto de las remociones de biomasa a los 15 años post-aprovechamiento. Sin embargo, Merino et al. (1998) afirmó que, es de esperar que se presente un aumento en los niveles de nitrógeno post-aprovechamiento, e incluso que podría retornar a niveles originales luego de tres años. Adicionalmente, Powers et al. (2005) aseguraron que hay una disminución en las concentraciones de nitrógeno en sitios donde se removió la biomasa, que afectará el nitrógeno disponible y mineralizable, y los niveles de nitrógeno foliar, principalmente en sitios con baja productividad.

Desde el punto de vista de la cantidad de nitrógeno para segundos aprovechamientos y según la metodología de Merino et al. (2005) se calculó que en los residuos del aprovechamiento, habrían alcanzado valores de: 84,4 $\mathrm{kg} / \mathrm{ha}$ de nitrógeno en ramas, $20,9 \mathrm{~kg} / \mathrm{ha}$ de nitrógeno en corteza y 106,2 kg/ha de nitrógeno en follaje; además de $186,0 \mathrm{~kg} / \mathrm{ha}$ de nitrógeno en fuste, los cuales fueron principalmente exportados en las trozas extraídas. Sin embargo, el nitrógeno total del suelo de los primeros $10 \mathrm{~cm}$ fue mayor a $1000 \mathrm{~kg} /$ ha para cualquiera de los tratamientos en el 2008, lo que se puede interpretar como una reserva adecuada para una segunda rotación.

Modelado de la dinámica del carbono en el suelo. El modelo utilizado también por Piñeiro et al. (2008) para describir la dinámica del carbono del suelo en el primer año post- aprovechamiento, permitió proyectar la caída de los contenidos de carbono lábil, la cual alcanzó pérdidas de más de $40 \%$ del valor inicial entre los meses de noviembre a enero de los dos años de mediciones. El modelo logró explicar, desde el punto de vista biológico, el comportamiento del carbono en el suelo para el período del primer año post- aprovechamiento. Al adicionar a este modelo un componente sinusoidal, se logró mejorar la explicación de la varianza que se dio en el tiempo con el carbono del suelo. Al modelar los tratamientos individualmente, se detectaron diferencias entre el tratamiento $\mathrm{X} 0$ con los tratamientos $\mathrm{X} 1$ y $\mathrm{X} 2$. Estas diferencias se podrían explicar por el efecto en las condiciones microclimáticas que modifica la presencia de los residuos del aprovechamiento sobre el suelo. Sin embargo, las diferencias microclimáticas no fueron evaluadas en este caso.

\section{Conclusiones}

La manipulación de los residuos del aprovechamiento forestal no afectó la dinámica de los contenidos de carbono en el suelo.

Al duplicar la cantidad de materia orgánica sobre el suelo, se aumentó la biomasa del horizonte orgánico 
Oe no manipulado. También se evidenció un descenso significativo de los desechos orgánicos entre el primer y segundo año post- aprovechamiento. Estos cambios no afectaron los niveles de carbono de los primeros $10 \mathrm{~cm}$ en el suelo, como consecuencia se podría aumentar los niveles de $\mathrm{CO}_{2}$ atmosférico.

Los horizontes orgánicos Oi y Oe presentaron el mayor descenso en los contenidos de biomasa en el primer año post- aprovechamiento, sin embargo, estos cambios no afectaron los contenidos de carbono en el suelo.

La dinámica del carbono del suelo fue explicada en un 84,7 \% por medio de un modelo de decaimiento cinético de primer orden. El modelo evaluado permitió evidenciar diferencias entre los tratamientos. Sin embargo, estas diferencias fueron en magnitud y no en la dinámica del carbono en el suelo.

\section{Agradecimientos}

Al Sr. Luis Castro por su colaboración en los muestreos y análisis de las muestras, a Simón Sandoval y Manuel Acevedo por sus aportes al manuscrito, a la Cooperativa de Nutrición Forestal por el financiamiento de algunos análisis.

\section{Referencias}

Brady, N. \& Weil, R. (2002). Elements of the nature and properties of soil. $13^{\text {th }}$ edition (606 p.). New Jersey, USA: Pearson Prentice Hall.

Brais, S., Paré, D., Camiré, C., Rochon, P. \& Vasseur, C. (2002). Nitrogen net mineralization and dynamics following whole-tree harvesting and winter windrowing on clayey sites of northwestern Quebec. Forest Ecology and Management, 157, 119-130.

Esquivel, E., Rubilar, R., Sandoval, S., Acuña, E., Cancino, J., Espinosa, M., \& Muñoz, F. (2013). Efecto de plantaciones dendroenergéticas en el carbono a nivel de suelo, en dos suelos contrastantes de la región de Biobío, Chile. Revista Árvore, 37(6), 1135-1144.

Fisher, R. \& Binkley, D. (1999). Ecology and management of forest soil. $3^{\text {rd }}$ edition (489 p.). New York, USA: John Wiley and Sons, INC.

Fleming, R., Powers, R., Foster, N., Kranabetter, J., Scott, D., Ponder, F ... Tiarks, A. (2006). Effects of organic matter removal, soil compaction, and vegetation control on 5-year seedling performance: a regional comparison of Long-Term Soil Productivity sites. Canadian Journal Forest Research, 36, 529-550.

Gerding, V. y Schlatter, J. (1999). Estabilidad nutritiva de plantaciones de Pinus radiata D. Don en cinco sitios caracterizados de la VIII Región. Bosque, 20(2), 107-115.

Huang, Z., Xu, Z., Chen, C. \& Boyd, S. (2008). Change in soil carbon during the establishment of hardwood plantation in subtropical Australia. Forest Ecology and Management, $254,46-55$.
Jurgensen, M., Harvey, A., Grahan, R., Page-Dumroese, D., Tonn, J., Larsen, M. \& Jain, T. (1997). Impacts of timber harvesting on soil organic matters, nitrogen, productivity, and health of Inland northwest Forest. Forest Science, 43(2), 234-251.

Kabzems, R. \& Haeussler, S. (2005). Soil properties, aspen, and white spruce responses 5 year after organic matter removal and compaction treatments. Canadian Journal Forests Research, 35, 2045-2055.

Kimmins, J. (1995). Forest ecology. $2^{\text {nd }}$ edition (596 p.). New Jersey, USA: Prentice-hall, Inc.

Longeri, L., Etchevers, J. y Venegas, J. (1979). Metodología de perfusión para estudios de nitrificación en suelos. Ciencia e Investigación Agraria, 6, 295-299.

Magdoff, F. (1996). Soil organic matter fractions and implications for interpreting organic matter test. In Magdoff F, M Tabatabai, Jr E Hanlon (Eds.) Soil organic matter: analysis and interpretation. SSSA Special Publication $\mathrm{N}^{\circ} 46$. Wisconsin, USA. Soil Science Society of America Inc. p. 11-20.

Mathers, N., Mendham, D., Connell, O` AM., Grove, T., Xu, Z. \& Saffigna, P. (2003). How does residue management impact soil organic matter composition and quality Eucalyptus globulus plantation in southwestern Australia?. Forest Ecology and Management, 179, 253-267.

Merino, A., Edeso, J., González, M. \& Marauri, P. (1998). Soil properties in a hilly area following different harvesting management practices. Forest Ecology and Management, 103, 235-246.

Merino, A., Rey, C., Brañas, J. y Rodríguez-Soalleiro, R. (2003). Biomasa arbórea y acumulación de nutrientes en plantaciones de Pinus radiata D. Don en Galicia. Investigación Agraria, Sistema y Recursos Forestales, 12(2), 85-98.

Merino, A., Balboa, M., Rodríguez-Solleiro, R. \& ÁlvarezGonzález, R. (2005). Nutrient exports under different harvesting regimes in fast-growing forest plantations in southern Europe. Forest Ecology and Management, 207, 325-339.

Observatorio Geodésico TIGO. (2008). Datos meteorológicos. Recuperado de http://www.tigo.cl/index. php?option=com_tigometeo\&ltemid=195\&lang=es

Piñeiro, G., Jobbágy, E., Rizzotto, M. \& Jackson, R. (2008). First order decay models fail to represent soil organic carbon dynamics in both single-pool and in multiple-pool models based on size fractionation. American Geophysical Union. Recuperado de http://adsabs.harvard.edu/ abs/2008AGUFM.B12A..08P

Powers, R. (1999). On the sustainable productivity of planted forest. New Forests, 17, 236-306.

Powers, R., Scott, D., Sánchez, F., Voldseth, R., Page-Dumroese, D., Elioff, D. \& Stone, D. (2005). The North American longterm soil productivity experiment: Findings from the first decade of research. Forest Ecology and Management, $220,31-50$.

Rubilar, R. (2003). Biomass and nutrient accumulation comparison between successive loblolly pine rotations on the upper coastal plain of Alabama. (Tesis Magíster en Ciencias). Department of Forestry, North Carolina State University: Raleigh, USA. 
Sánchez, G., Tiarks, A., Kranabetter, J., Page-Dumroese, D., Powers, R., Sanborn, P. \& Chapman, W. (2006). Effects of organic matter removal and soil compaction on fifth-year mineral soil carbon and nitrogen contents for sites across the United States and Canada. Canadian Journal Forests Research, 36, 565-576.

Sparks, D. (1995). Environmental Soil Chemistry. (267 p.) .California, USA: Academic Press, INC.

SAS-Institute-Inc. (2000). SAS/STAT User's Guide: Version 8, vol. 1, 2 and 3. SAS Institute Inc., NC, U.S.A.

SAS Institute Inc. (2008). JMP 8 User Guide. Cary, NC: SAS Institute Inc. U.S.A.

Schoenholtz, S., Van Miegroet, H. \& Burger, J. (2000). A review of chemical and physical properties as indicators of forest soil quality: challenges and opportunities. Forest Ecology and Management, 138, 335-356.

Tabatabai, M. (1996). Soil organic matter testing: an overview. In FR. Magdoff, MA Tabatabai \& Jr.EA Hanlon (Eds.) Soil organic matter: analysis and interpretation. SSSA Special Publication $\mathrm{N}^{\circ}$. 46. (pp. 1-9). Wisconsin, USA: Soil Science Society of America, Inc.

Ussiri, D. \& Johnson, C. (2007). Organic matter composition and dynamics in a northern hardwood forest ecosystem 15 years after clear-cutting. Forest Ecology and Management, 240, 131-142.

USDA (United States Departament of Agriculture). (2006). Soil Taxonomy Handbook 436. Washington, DC: Soil Cons. Serv.

Vitousek, P. (1985). Disturbance, nitrogen availability, and nitrogen losses in an intensively managed Loblolly Pine plantation. Ecology, 66(4), 1360 - 1376.

Waring, R. \& Running, S. (1998). Forest ecosystems analysis at multiples scales. $2^{\text {nd }}$ edition. (370 p.). USA: Academic Press.

Weisberg, S. (1985). Applied linear regression. $2^{\text {nd }}$ edition. (324 p.). USA: Wiley.

Ximenes, F., Gardener, W. \& Kathuria, A. (2008). Proportion of above-ground biomass in commercial logs and residues following the harvest of five commercial forest species in Australia. Forest Ecology and Management, 256, 335346.

Yanai, R., Arthur, M., Siccama, T. \& Federer, C. (2000). Challenges of measuring forest floor organic matter dynamics: repeated measures from a chronosequence. Forest Ecology and Management, 138, 273-283.

Yanai, R., Currier, W. \& Goodale, C. (2003). Soil carbon dynamics after forest harvest: an ecosystem paradigm reconsidered. Ecosystems, 6, 197-212. 